\title{
HOMOGENIZATION OF OBSTACLE PROBLEMS IN ORLICZ-SOBOLEV SPACES
}

\author{
DIEGO MARCON, JOSÉ FRANCISCO RODRIGUES, \\ AND RAFAYEL TEYMURAZYAN
}

\begin{abstract}
We study the homogenization of obstacle problems in OrliczSobolev spaces for a wide class of monotone operators (possibly degenerate or singular) of the $p(\cdot)$-Laplacian type. Our approach is based on the Lewy-Stampacchia inequalities, which then give access to a compactness argument. We also prove the convergence of the coincidence sets under non-degeneracy conditions.
\end{abstract}

Keywords: Homogenization, obstacle problem, Orlicz-Sobolev spaces, convergence of coincidence sets.

AMS Subject Classifications MSC 2010: 35J20, 35J62, 35J92, 35D30, 35A 15 .

\section{INTRODUCTION}

Let $\Omega \subset \mathbb{R}^{n}$ be a bounded domain and $p: \Omega \rightarrow \mathbb{R}$ be measurable and such that

$$
1<\alpha \leq p(x) \leq \beta<\infty \text { a.e. in } \Omega,
$$

where $\alpha$ and $\beta$ are constants. The following variable exponent Lebesgue space is an Orlicz space:

$$
L^{p(\cdot)}(\Omega):=\left\{u: \Omega \rightarrow \mathbb{R} \text { measurable } \rho(u):=\int_{\Omega}|u(x)|^{p(x)} d x<\infty\right\} .
$$

This Orlicz space is a separable reflexive Banach space with the following (Luxembourg) norm:

$$
\|u\|_{L^{p(\cdot)}(\Omega)}:=\inf \left\{\lambda>0, \rho\left(\frac{|u|}{\lambda}\right) \leq 1\right\} .
$$

We define an Orlicz-Sobolev space by

$$
W^{1, p(\cdot)}(\Omega):=\left\{u \in L^{p(\cdot)}(\Omega), \nabla u \in\left(L^{p(\cdot)}(\Omega)\right)^{n}\right\},
$$

with the norm

$$
\|u\|_{W^{1, p(\cdot)}(\Omega)}:=\|u\|_{L^{p(\cdot)}(\Omega)}+\|\nabla u\|_{L^{p(\cdot)}(\Omega)},\|\nabla u\|_{L^{p(\cdot)}(\Omega)}=\sum_{i=1}^{n}\left\|\frac{\partial u}{\partial x_{i}}\right\|_{L^{p(\cdot)}(\Omega)} .
$$


This Orlicz-Sobolev space is also a separable and reflexive Banach space. We also define

$$
W_{0}^{1, p(\cdot)}(\Omega):=\left\{u \in W_{0}^{1,1}(\Omega), \rho(|\nabla u|)<\infty\right\} .
$$

The latter is a Banach space endowed with the norm

$$
\|u\|_{W_{0}^{1, p(\cdot)}(\Omega)}:=\|\nabla u\|_{L^{p(\cdot)}(\Omega)} \cdot
$$

In this paper we study the periodic homogenization of obstacle problems in Orlicz-Sobolev spaces. We consider

$$
a(x, \xi): \Omega \times \mathbb{R}^{n} \rightarrow \mathbb{R}^{n}
$$

to be a Carathéodory vector function, that is, we assume it is continuous with respect to $\xi$, for almost every $x \in \mathbb{R}^{n}$, and that it is measurable with respect to $x$, for every $\xi$. Moreover, the functions $a(\cdot, \xi)$ and $p(\cdot)$ are assumed to be periodic with period 1 in each argument $x_{1}, x_{2}, \ldots, x_{n}$. We denote the periodicity cell by $Q$, i.e. $Q:=(0,1]^{n}$. Additionally, we assume that the following structural conditions (monotonicity, coercitivity and boundedness) hold:

$$
\left\{\begin{array}{l}
(a(x, \xi)-a(x, \eta)) \cdot(\xi-\eta)>0, \text { for a.e. } x, \xi \neq \eta \\
a(x, \xi) \cdot \xi \geq C_{1}\left(|\xi|^{p(x)}-1\right), \\
|a(x, \xi)| \leq C_{2}\left(|\xi|^{p(x)-1}+1\right)
\end{array}\right.
$$

where $C_{1}, C_{2}>0$ are constants. For $\varepsilon>0$, we define

$$
a_{\varepsilon}(x, \xi):=a\left(\frac{x}{\varepsilon}, \xi\right), x \in \Omega, \xi \in \mathbb{R}^{n}
$$

and $p_{\varepsilon}(x)=p(x / \varepsilon)$. The Orlicz-Sobolev spaces of periodic functions, denoted by $W_{\text {per }}^{1, p(\cdot)}(Q)$, is defined as the set of periodic functions $u$ from $W_{\text {per }}^{1,1}(Q)$ with

$$
\int_{Q} u d x=0 \quad \text { and } \quad \int_{Q}|\nabla u|^{p(x)} d x<\infty .
$$

For the homogenized functional defined by

$$
h(\xi):=\min _{v \in W_{\mathrm{per}}^{1, p(\cdot)}(Q)} \int_{Q} \frac{|\xi+\nabla v|^{p(x)}}{p(x)} d x,
$$

we introduce also the Orlicz-Sobolev spaces

$$
\begin{aligned}
W^{h}(\Omega) & :=\left\{u \in W^{1,1}(\Omega), h(\nabla u) \in L^{1}(\Omega)\right\}, \\
W_{0}^{h}(\Omega) & :=\left\{u \in W_{0}^{1,1}(\Omega), h(\nabla u) \in L^{1}(\Omega)\right\},
\end{aligned}
$$

with the norm, $\|u\|_{W_{0}^{h}(\Omega)}:=\|\nabla u\|_{L^{h}(\Omega)}$, and the vector Orlicz space

$$
L^{h}(\Omega):=\left\{\xi \in\left[L^{1}(\Omega)\right]^{n}, h(\xi) \in L^{1}(\Omega)\right\},
$$

normed by

$$
\|\xi\|_{L^{h}(\Omega)}:=\inf \left\{\lambda>0, \int_{\Omega} h\left(\frac{\xi}{\lambda}\right) \leq 1\right\} .
$$


By the properties of $h$, as it was observed in [24], we have the continuous embeddings

$$
L^{\beta}(\Omega) \subset L^{h}(\Omega) \subset L^{\alpha}(\Omega),
$$

Assuming that

$$
\begin{gathered}
f \text { and }\left(A_{\varepsilon} \psi_{\varepsilon}-f\right)^{+} \in L^{s}(\Omega), \\
\left\|\left(A_{\varepsilon} \psi_{\varepsilon}-f\right)^{+}\right\|_{L^{s}(\Omega)} \leq C,
\end{gathered}
$$

where $C>0$ is a constant independent of $\varepsilon$ and

$$
\psi_{\varepsilon} \in W^{1, p_{\varepsilon}(\cdot)}(\Omega), \psi_{0} \in W^{h}(\Omega), \psi_{\varepsilon}^{+} \in W_{0}^{1, p_{\varepsilon}(\cdot)}(\Omega), \psi_{0}^{+} \in W_{0}^{h}(\Omega),
$$

where $\alpha^{\prime}=\alpha /(\alpha-1), u^{+}$is the positive part of $u$ and $s>\frac{n \alpha^{\prime}}{n+\alpha^{\prime}}$ if $\alpha<n$, $s>1$, if $\alpha=n$ and $s=1$ for $\alpha>n$, we show (Theorem 3.1) that the unique solution $u_{\varepsilon} \in K_{\varepsilon}$ of the obstacle problem

$$
\int_{\Omega} a_{\varepsilon}\left(x, \nabla u_{\varepsilon}\right) \cdot \nabla\left(v-u_{\varepsilon}\right) d x \geq \int_{\Omega} f\left(v-u_{\varepsilon}\right) d x, \quad \forall v \in K_{\varepsilon},
$$

where

$$
K_{\varepsilon}:=\left\{v \in W_{0}^{1, p_{\varepsilon}(\cdot)}(\Omega), v \geq \psi_{\varepsilon} \text { a.e. in } \Omega\right\},
$$

converges to the unique solution $u_{0} \in K_{0}$ of the following homogenized obstacle problem

$$
\int_{\Omega} a_{0}\left(\nabla u_{0}\right) \cdot \nabla\left(v-u_{0}\right) d x \geq \int_{\Omega} f\left(v-u_{0}\right) d x, \quad \forall v \in K_{0},
$$

where

$$
K_{0}:=\left\{v \in W_{0}^{h}(\Omega), v \geq \psi_{0} \text { a.e. in } \Omega\right\} .
$$

The homogenized operator $a_{0}: \mathbb{R}^{n} \rightarrow \mathbb{R}^{n}$ is given in terms of the weighted average of $a$ as in [24], that is,

$$
a_{0}(\xi):=\int_{Q} a(x, \xi+\nabla v) d x
$$

with $v \in W_{\text {per }}^{1, p(\cdot)}(Q)$, such that,

$$
\int_{Q} a(x, \xi+\nabla v) \cdot \nabla \varphi d x=0, \quad \forall \varphi \in W_{\mathrm{per}}^{1, p(\cdot)}(Q),
$$

where $Q$ is the periodicity cell.

Note that, due to the Lavrent'ev effect, if instead of $W_{\text {per }}^{1, p(\cdot)}(Q)$, we take $\varphi \in C_{\text {per }}^{\infty}(Q)$, we may end up with a different homogenized operator, since in general the space $C_{\mathrm{per}}^{\infty}(Q)$ is not dense in $W_{\mathrm{per}}^{1, p(\cdot)}(\Omega)$. These homogenized operators, referred to as $W$ and $H$ solutions in [24], respectively, in general may be different, but our results hold for both solutions, with minor modifications for the space framework of the $H$ solutions. Although we prefer to work with $W$ solutions, that is due to the fact that [24, Theorem 3.1] (see Theorem 2.1 below) is true for both types of solutions. Observe that we do 
not impose any regularity assumption on $p(\cdot)$. However, in the particular case when $p$ is $\log$-Lipschitz continuous, i.e., when for a constant $L>0$

$$
-|p(x)-p(y)| \log |x-y| \leq L, \quad \forall x, y \in \bar{\Omega},|x-y|<1 / 2,
$$

the notion of $W$ and $H$ solutions coincide (see $[10,14]$ ), since then the smooth functions are dense in the Orlicz-Sobolev space.

Our approach is a development of the classical methods $[6,11]$ (see also $[20,21,24])$ combined with the Lewy-Stampacchia inequalities in the OrliczSobolev framework, in accordance with [19], which then allows the use of a Rellich-Kondrachov compactness argument.

The result generalizes, in part, that of [5], which covers the case when $p$ is constant (and hence the homogenization is in usual Sobolev spaces). The latter, in turn, implies the case of $p=2$ obtained in [4]. Nonetheless, we observe that the structural assumptions (1.2) allow us to consider a wider range of monotone operators, which cover these cases and include other interesting quasilinear operators, some of which we list below.

1. If $a(x, \xi)=|\xi|^{p(x)-2} \xi$, we deal with the obstacle problem for the $p(x)$ Laplace operator.

2. We can also consider perturbations of the $p$-Laplace ( $p$ constant) and of the $p(x)$-Laplace operators, taking

$$
a(x, \xi)=\gamma(x)|\xi|^{p-2} \xi \text { and } a(x, \xi)=\gamma(x)|\xi|^{p(x)-2} \xi
$$

for any non-negative bounded periodic function $\gamma(x)$.

3 . It is possible to consider functions which are essentially different from these previous "power like" functions. One general example can be

$$
a(x, \xi)=\gamma_{1}(x)|\xi|^{p(x)-1} \xi \log \left(\gamma_{2}(x)|\xi|+\gamma_{3}(x)\right),
$$

where $\gamma_{3}(x), p(x)>1$ and $\gamma_{1}(x), \gamma_{2}(x)>0$ a.e. in $\Omega$ are bounded periodic functions.

The paper is organized as follows: in Section 2, we state some preliminaries facts, which then serve to prove our main result in Section 3 (Theorem 3.1). In Section 4, we prove the convergence of the coincidence sets (Theorems 4.1 and 4.2).

\section{Preliminaries}

In this section we give some preliminaries. In particular, we provide the concept of $G$-convergence of operators in our framework, as well as convergence of sets in Mosco sense. We also recall some results from [22] and [24] for future reference. We start by setting some notations, which will be used throughout the paper: $p_{\varepsilon}(x)=p(x / \varepsilon) ; \alpha^{\prime}=\frac{\alpha}{\alpha-1} ; \rightarrow$ denotes the weak convergence;

$$
A_{\varepsilon} u:=-\operatorname{div}\left(a_{\varepsilon}(x, \nabla u)\right) \text { and } A_{0} u:=-\operatorname{div}\left(a_{0}(\nabla u)\right),
$$


where $a_{\varepsilon}$ is defined by (1.3), and $a_{0}$ is defined by (1.10). Next, we define the notion of $G$-convergence of $a_{\varepsilon}$ to $a_{0}$. Observe, that most definitions of $G$-convergence that can be found in the literature (see, for example, $[2,3,7,17])$, allow $a_{0}$ to depend on $x$ as well, just as $a_{\varepsilon}$ depends. However, in some particular cases, more information can be said about the limiting operator. One example is that of operators with rapidly oscillating "coefficients". Since our assumptions ensure that $a(x, \xi)$ and $p(\cdot)$ are periodic with respect to $x$ in each of the arguments $x_{1}, x_{2}, \ldots, x_{n}$, there is no loss in generality to impose $a_{0}$ to be independent of $x$ in the definition of $G$-convergence, which is more relevant for our purposes.

Definition 2.1. Consider $a_{\varepsilon}: \Omega \times \mathbb{R}^{n} \rightarrow \mathbb{R}^{n}$ and $a_{0}: \mathbb{R}^{n} \rightarrow \mathbb{R}^{n}$ as above. We say that $a_{\varepsilon} G$-converges to $a_{0}$ when, considering the unique solution $u_{\varepsilon} \in W_{0}^{1, p_{\varepsilon}(\cdot)}(\Omega)$ of

$$
-\operatorname{div}\left(a_{\varepsilon}\left(x, \nabla u_{\varepsilon}\right)\right)=f, f \in W_{0}^{-1, \alpha^{\prime}}(\Omega) \text { in } \mathcal{D}^{\prime}(\Omega)
$$

and $u_{0} \in W_{0}^{h}(\Omega)$ the unique solution of

$$
-\operatorname{div}\left(a_{0}\left(\nabla u_{0}\right)\right)=f \quad \text { in } \mathcal{D}^{\prime}(\Omega)
$$

there holds:

(1) $u_{\varepsilon} \rightarrow u_{0}$ in $W_{0}^{1, \alpha}(\Omega)$, as $\varepsilon \rightarrow 0$;

(2) $a_{\varepsilon}\left(x, \nabla u_{\varepsilon}\right) \rightarrow a_{0}\left(\nabla u_{0}\right)$ in $\left(L^{\beta^{\prime}}(\Omega)\right)^{n}$, as $\varepsilon \rightarrow 0$.

Note that the choice of $s$ in (1.5) guarantees, in particular, $f \in W^{-1, \alpha^{\prime}}(\Omega)$. Additionally, $a(x, \xi)$ is assumed to be continuous with respect to $\xi$, for almost every $x \in \mathbb{R}^{n}$.

Next, we state a theorem from [24, Theorem 3.1] that insures the $G$ convergence of $a_{\varepsilon}$ to a function $a_{0}$, as $\varepsilon \rightarrow 0$, given explicitly in terms of $a$. Its proof is based on a compensated compactness argument from [23, 24], which, in the case of $p(\cdot)=$ constant, resembles the well known result of Tartar-Murat (see [16]).

Theorem 2.1. Let $a(x, \xi)$ be a Carathéodory vector function, which is periodic with respect to $x$ in each argument and satisfy (1.2). Let also $p$ be periodic, measurable and satisfy (1.1). If structural conditions (1.2) hold, then $a_{\varepsilon} G$-converges to $a_{0}$, where $a_{0}$ is defined by (1.10). Moreover,

$$
\int_{\Omega} a_{\varepsilon}\left(x, \nabla u_{\varepsilon}\right) \cdot \nabla u_{\varepsilon} d x \rightarrow \int_{\Omega} a_{0}\left(\nabla u_{0}\right) \cdot \nabla u_{0} d x,
$$

as $\varepsilon \rightarrow 0$.

As it is shown in [24], the vector function $a_{0}(\xi)$ is strictly monotone, i.e.,

$$
\left(a_{0}(\xi)-a_{0}(\eta)\right) \cdot(\xi-\eta)>0, \quad \xi \neq \eta,
$$

and coercive, that is,

$$
a_{0}(\xi) \cdot \xi>c_{0}(h(\xi)-1),
$$


where $c_{0}>0$ is a constant, and the homogenized functional $h(\xi)$ is defined by (1.4). Moreover, $h$ satisfies the so-called $\Delta_{2}$ condition, [24, Proposition 2.1 , which implies that the Orlicz space $L^{h}(\Omega)$ is reflexive. As it is observed in [24], $h(\xi)$ being defined by (1.4), is convex on $\mathbb{R}^{n}$ and satisfies the following two-sided estimate:

$$
c_{1}|\xi|^{\alpha}-1 \leq h(\xi) \leq c_{2}|\xi|^{\beta}+1,
$$

for a $c_{1}>0$ constant. As a consequence, we have

$$
W_{0}^{1, \beta}(\Omega) \subset W_{0}^{h}(\Omega) \subset W_{0}^{1, \alpha}(\Omega),
$$

which implies that

$$
K_{0} \subset W_{0}^{1, \alpha}(\Omega) .
$$

The following result is from [22], and it provides more information on the homogenized functional.

Lemma 2.1. If $u_{\varepsilon}$ is a sequence uniformly bounded in $W_{0}^{1, p_{\varepsilon}(\cdot)}(\Omega)$, such that, $u_{\varepsilon} \rightarrow u_{0}$ in $W_{0}^{1, \alpha}(\Omega)$ as $\varepsilon \rightarrow 0$, then $h\left(\nabla u_{0}\right) \in L^{1}(\Omega)$.

Observe that Lemma 2.1 guarantees that, within $G$-convergence, the weak limits of $u_{\varepsilon}$ in $W_{0}^{1, \alpha}(\Omega)$ belong to $W_{0}^{h}(\Omega)$, and therefore, if also $u_{\varepsilon} \in K_{\varepsilon}$ then $u_{0} \in K_{0}$.

In order to state our main result, we will also need to redefine the Mosco convergence of sets.

Definition 2.2. The sequence of closed convex sets $K_{\varepsilon} \subset W_{0}^{1, p_{\varepsilon}(\cdot)}(\Omega)$, is said to converge to the set $K_{0} \subset W_{0}^{h}(\Omega)$ in the Mosco sense, if

- for any $v_{0} \in K_{0}$ there exists a sequence $v_{\varepsilon} \in K_{\varepsilon}$, such that, $v_{\varepsilon} \rightarrow v_{0}$ in $W_{0}^{1, \alpha}(\Omega)$;

- weak limits in $W_{0}^{1, \alpha}(\Omega)$ of any sequence of elements in $K_{\varepsilon}$, that is uniformly bounded in $W_{0}^{1, p_{\varepsilon}(\cdot)}(\Omega)$, belong to $K_{0}$.

Remark 2.1. Since $W_{0}^{1, p_{\varepsilon}(\cdot)}(\Omega)$ is continuously embedded into $W_{0}^{1, \alpha}(\Omega)$ (see, for example, [10]), then $\psi_{\varepsilon} \rightarrow \psi_{0}$ in $W^{1, \beta}(\Omega)$ provides $K_{\varepsilon} \rightarrow K_{0}$ in the Mosco sense, where $K_{\varepsilon}$ and $K_{0}$ are as in (1.8) and (1.9) respectively.

\section{Homogenization of the obstacle PRoblem}

We are now ready to prove our main result, which states as follows.

Theorem 3.1. Let $a(x, \xi)$ be a Carathéodory vector function satisfying (1.2) and periodic with respect to $x$ in each argument. Let $p(\cdot)$ be periodic, measurable and satisfying (1.1). Assume further that (1.5)-(1.7) hold. If $K_{\varepsilon} \rightarrow K_{0}$ in the Mosco sense, then the unique solution of (1.8) converges weakly in $W_{0}^{1, \alpha}(\Omega)$, as $\varepsilon \rightarrow 0$, to the unique solution of (1.9), where $a_{0}$ is given by $(1.10)$. 
Proof. We divide the proof into five steps.

Step 1 (Apriori estimates). Existence and uniqueness of the solution of (1.8) (and (1.9)) is a classical result (see, for instance, $[9,18,19])$. As in the proof of [5, Theorem 2.3] (see also [18, page 145]), the coercitivity and boundedness assumptions from (1.2) imply that $u_{\varepsilon}$ is bounded in $W_{0}^{1, p_{\varepsilon}(\cdot)}(\Omega)$ by a constant depending only from $C_{1}, C_{2}$ but independent of $\varepsilon$. For the details we refer the reader to [12]. As a consequence we obtain that $u_{\varepsilon}$ is bounded also in $W_{0}^{1, \alpha}(\Omega)$, since $W_{0}^{1, p_{\varepsilon}(\cdot)}(\Omega) \subset W_{0}^{1, \alpha}(\Omega)$. Set

$$
\sigma_{\varepsilon}:=a_{\varepsilon}\left(x, \nabla u_{\varepsilon}\right), \quad \mu_{\varepsilon}:=-\operatorname{div}\left(a_{\varepsilon}\left(x, \nabla u_{\varepsilon}\right)\right)-f .
$$

The boundedness condition from (1.2) implies that $\sigma_{\varepsilon}$ and $\mu_{\varepsilon}$ are bounded (see $[5,24]$ ), therefore we can extract weakly convergent subsequence (still denoted by $\varepsilon$ ) from each one of them. Thus, there exist $u^{*}, \sigma^{*}, \mu^{*}$ such that

$$
\begin{gathered}
u_{\varepsilon} \rightarrow u^{*} \quad \text { in } W_{0}^{1, \alpha}(\Omega) \text { and } u_{\varepsilon} \rightarrow u^{*} \text { in } L^{\alpha}(\Omega), \\
\sigma_{\varepsilon} \rightarrow \sigma^{*} \text { in }\left(L^{\beta^{\prime}}(\Omega)\right)^{n}, \\
\mu_{\varepsilon} \rightarrow \mu^{*} \text { in } W^{-1, \beta^{\prime}}(\Omega) .
\end{gathered}
$$

Note that

$$
\mu^{*}=-\operatorname{div} \sigma^{*}-f .
$$

Moreover, using Lemma 2.1 and since $K_{\varepsilon} \rightarrow K_{0}$ in the Mosco sense, then

$$
u^{*} \in K_{0} \text {. }
$$

Step 2 (Compactness). Note that our assumptions provide the LewyStampacchia inequalities (see [19]), that is, we have

$$
f \leq f+\mu_{\varepsilon} \leq\left(A_{\varepsilon} \psi_{\varepsilon}-f\right)^{+}+f,
$$

which implies, by a Rellich-Kondrachov compactness argument,

$$
\mu_{\varepsilon} \rightarrow \mu^{*} \text { in } W^{-1, \alpha^{\prime}}(\Omega) .
$$

Step 3. In this step we prove that $\sigma^{*}=a_{0}\left(\nabla u^{*}\right)$, where $a_{0}$ is defined by (1.10). To see this, let $w_{0} \in \mathcal{D}(\Omega)$ and $w_{\varepsilon} \in W_{0}^{1, p_{\varepsilon}(\cdot)}(\Omega)$ be the unique solution of

$$
\operatorname{div}\left(a_{\varepsilon}\left(x, \nabla w_{\varepsilon}\right)\right)=\operatorname{div}\left(a_{0}\left(\nabla w_{0}\right)\right) \quad \text { in } \mathcal{D}^{\prime}(\Omega) .
$$

From Theorem 2.1, we have that $a_{\varepsilon} G$-converges to $a_{0}$, as $\varepsilon \rightarrow 0$, where $a_{0}(\xi)$ is defined by (1.10). In particular,

$$
\left\{\begin{array}{l}
w_{\varepsilon} \rightarrow w_{0} \text { in } W^{1, \alpha}(\Omega) \\
a_{\varepsilon}\left(x, \nabla w_{\varepsilon}\right) \rightarrow a_{0}\left(\nabla w_{0}\right) \text { in }\left(L^{\beta^{\prime}}(\Omega)\right)^{n} .
\end{array}\right.
$$

Fix now $\varphi$ such that

$$
\varphi \in \mathcal{D}(\Omega), \quad 0 \leq \varphi \leq 1
$$

From the monotonicity of $a_{\varepsilon}$ one has

$$
\int_{\Omega} \varphi\left(a_{\varepsilon}\left(x, \nabla u_{\varepsilon}\right)-a_{\varepsilon}\left(x, \nabla w_{\varepsilon}\right)\right) \cdot\left(\nabla u_{\varepsilon}-\nabla w_{\varepsilon}\right) d x \geq 0 .
$$


Since $u^{*} \in K_{0}$, and $K_{\varepsilon} \rightarrow K_{0}$ in the Mosco sense, there exists a sequence $\bar{u}_{\varepsilon}$, such that,

$$
\bar{u}_{\varepsilon} \in K_{\varepsilon} \quad \text { and } \quad \bar{u}_{\varepsilon} \rightarrow u^{*} \text { in } W_{0}^{1, \alpha}(\Omega) .
$$

Next, we write (3.11) as

$$
\begin{aligned}
& \int_{\Omega} \varphi \sigma_{\varepsilon} \cdot\left(\nabla u_{\varepsilon}-\nabla \bar{u}_{\varepsilon}\right)+\int_{\Omega} \varphi \sigma_{\varepsilon} \cdot \nabla \bar{u}_{\varepsilon}-\int_{\Omega} \varphi \sigma_{\varepsilon} \cdot \nabla w_{\varepsilon} \\
&-\int_{\Omega} \varphi a_{\varepsilon}\left(x, \nabla w_{\varepsilon}\right) \cdot \nabla\left(u_{\varepsilon}-w_{\varepsilon}\right) \\
&:=I_{1}+I_{2}+I_{3}+I_{4} .
\end{aligned}
$$

Since $0 \leq \varphi \leq 1$ on $\Omega$, and $K_{\varepsilon}$ is convex, then the function $v=\varphi \bar{u}_{\varepsilon}+(1-\varphi) u_{\varepsilon}$ can be used as a test function in (1.8), which gives

$$
\int_{\Omega} \sigma_{\varepsilon} \cdot \nabla\left(\varphi\left(\bar{u}_{\varepsilon}-u_{\varepsilon}\right)\right) \geq \int_{\Omega} f \varphi\left(\bar{u}_{\varepsilon}-u_{\varepsilon}\right)
$$

and so

$$
\begin{aligned}
I_{1} & =\int_{\Omega} \sigma_{\varepsilon} \cdot \nabla\left(\varphi\left(u_{\varepsilon}-\bar{u}_{\varepsilon}\right)\right)-\int_{\Omega}\left(u_{\varepsilon}-\bar{u}_{\varepsilon}\right) \sigma_{\varepsilon} \cdot \nabla \varphi \\
& \leq \int_{\Omega} f \varphi\left(u_{\varepsilon}-\bar{u}_{\varepsilon}\right)-\int_{\Omega}\left(u_{\varepsilon}-\bar{u}_{\varepsilon}\right) \sigma_{\varepsilon} \cdot \nabla \varphi .
\end{aligned}
$$

Since $u_{\varepsilon}$ and $\bar{u}_{\varepsilon}$ converge to $u^{*}$ weakly in $W_{0}^{1, \alpha}(\Omega)$ (and strongly in $L^{\alpha}(\Omega)$ ), we obtain

$$
\limsup _{\varepsilon \rightarrow 0} I_{1} \leq 0
$$

As we know from (3.12), $\bar{u}_{\varepsilon} \rightarrow u^{*}$ in $W_{0}^{1, \alpha}(\Omega)$, which gives

$$
\lim _{\varepsilon \rightarrow 0} I_{2}=\int_{\Omega} \varphi \sigma^{*} \cdot \nabla u^{*}
$$

Note that

$$
I_{3}=-\int_{\Omega} \sigma_{\varepsilon} \cdot \nabla\left(\varphi w_{\varepsilon}\right)+\int_{\Omega} w_{\varepsilon} \sigma_{\varepsilon} \cdot \nabla \varphi .
$$

From (3.7) and (3.12), we pass to the limit in the first term of $I_{3}$. Using (3.3) and (3.12), we pass to the limit also in the second term of $I_{3}$, arriving at

$$
\lim _{\varepsilon \rightarrow 0} I_{3}=-\int_{\Omega} \varphi \sigma^{*} \nabla w_{0}
$$

Observe that

$$
I_{4}=-\int_{\Omega} a_{\varepsilon}\left(x, \nabla w_{\varepsilon}\right) \cdot \nabla\left(\varphi\left(u_{\varepsilon}-w_{\varepsilon}\right)\right)+\int_{\Omega}\left(u_{\varepsilon}-w_{\varepsilon}\right) a_{\varepsilon}\left(x, \nabla w_{\varepsilon}\right) \cdot \nabla \varphi,
$$

and recalling (3.2) and (3.9) and passing to the limit we obtain

$$
\lim _{\varepsilon \rightarrow 0} I_{4}=-\int_{\Omega} \varphi a_{0}\left(\nabla w_{0}\right) \cdot \nabla\left(u^{*}-w_{0}\right) .
$$


Combining (3.13), (3.15)-(3.18), one has

$$
\int_{\Omega} \varphi\left(\sigma^{*}-a_{0}\left(\nabla w_{0}\right)\right) \cdot \nabla\left(u^{*}-w_{0}\right) \geq 0 \quad \text { for } w_{0} \in \mathcal{D}(\Omega) .
$$

By density, (3.19) is true also for any $w_{0}$ in $W_{0}^{1, \alpha}(\Omega)$. Consider $w_{0}=u^{*}+t \varphi$, with $t \geq 0$ and $\varphi \in W_{0}^{1, \alpha}(\Omega)$. Letting $t \rightarrow 0$ and using Minty's trick as in [5, page 94] (see also [15]), we conclude

$$
\sigma^{*}=a_{0}\left(\nabla u^{*}\right)
$$

Step 4 (Lower semicontinuity of the energy). From (3.11) and (3.13) one has

$$
\begin{aligned}
\int_{\Omega} \varphi \sigma_{\varepsilon} \cdot \nabla u_{\varepsilon} & \geq \int_{\Omega} \varphi \sigma_{\varepsilon} \cdot \nabla w_{\varepsilon}+\int_{\Omega} \varphi a_{\varepsilon}\left(x, \nabla w_{\varepsilon}\right) \cdot \nabla\left(u_{\varepsilon}-w_{\varepsilon}\right) \\
& =-I_{3}-I_{4} .
\end{aligned}
$$

From $(3.17),(3.18)$ and $(3.20)$ for any $w_{0} \in \mathcal{D}(\Omega)$ we have

$$
\begin{aligned}
& \liminf _{\varepsilon \rightarrow 0} \int_{\Omega} \varphi \sigma_{\varepsilon} \cdot \nabla u_{\varepsilon} \\
\geq & \int_{\Omega} \varphi a_{0}\left(\nabla u^{*}\right) \cdot \nabla w_{0}+\int_{\Omega} \varphi a_{0}\left(\nabla w_{0}\right) \cdot \nabla\left(u^{*}-w_{0}\right) .
\end{aligned}
$$

Letting $w_{0}$ go to $u^{*}$ in $W_{0}^{1, \alpha}(\Omega)$, one gets from $(3.21)$

$$
\liminf _{\varepsilon \rightarrow 0} \int_{\Omega} \varphi \sigma_{\varepsilon} \cdot \nabla u_{\varepsilon} \geq \int \varphi a_{0}\left(\nabla u^{*}\right) \cdot \nabla u^{*},
$$

$\forall \varphi \in \mathcal{D}(\Omega)$ such that $0 \leq \varphi \leq 1$.

Step 5. Finally, we claim that $u^{*}$ is the unique solution $u_{0}$ of (1.9).

Let $v_{0} \in K_{0}$ and since $K_{\varepsilon} \rightarrow K_{0}$ in the Mosco sense, then there is a sequence $\bar{v}_{\varepsilon} \in K_{\varepsilon}$ such that $\bar{v}_{\varepsilon} \rightarrow v_{0}$ in $W_{0}^{1, \alpha}(\Omega)$. Using $\bar{v}_{\varepsilon}$ as a test function in (1.8) for $\varphi \in \mathcal{D}(\Omega), 0 \leq \varphi \leq 1$, one gets

$$
\int_{\Omega} \sigma_{\varepsilon} \cdot \nabla \bar{v}_{\varepsilon}-\int_{\Omega} f\left(\bar{v}_{\varepsilon}-u_{\varepsilon}\right) \geq \int_{\Omega} \sigma_{\varepsilon} \cdot \nabla u_{\varepsilon} \geq \int_{\Omega} \varphi\left(\sigma_{\varepsilon} \cdot \nabla u_{\varepsilon}\right) .
$$

Recalling (3.22) and passing to the limit in $\varepsilon$ in (3.23), we obtain

$$
\int_{\Omega} a_{0}\left(\nabla u^{*}\right) \cdot \nabla v_{0}-\int_{\Omega} f\left(v_{0}-u^{*}\right) \geq \int_{\Omega} \varphi a_{0}\left(\nabla u^{*}\right) \cdot \nabla u^{*} .
$$

Letting $\varphi \rightarrow 1$ in the last inequality, one gets

$$
\int_{\Omega} a_{0}\left(\nabla u^{*}\right) \cdot \nabla\left(v_{0}-u^{*}\right)-\int_{\Omega} f\left(v_{0}-u^{*}\right) \geq 0, \forall v_{0} \in K_{0} .
$$

The latter, combined with (3.6), allow us to conclude that $u^{*}$ coincides with the unique solution $u_{0}$ of (1.9) and the whole sequence $u_{\varepsilon} \rightarrow u_{0}$ in $W_{0}^{1, \alpha}(\Omega)$. 
Remark 3.1. One can also show the convergence of the energies. More precisely,

$$
\int_{\Omega} a_{\varepsilon}\left(x, \nabla u_{\varepsilon}\right) \cdot \nabla u_{\varepsilon} d x \rightarrow \int_{\Omega} a_{0}\left(\nabla u_{0}\right) \cdot \nabla u_{0} d x
$$

Proof. For any $\varphi \in \mathcal{D}(\Omega)$ such that $0 \leq \varphi \leq 1$ from (3.14) we have

$$
\int_{\Omega} \varphi \sigma_{\varepsilon} \cdot \nabla u_{\varepsilon} \leq \int_{\Omega} \sigma_{\varepsilon} \cdot \nabla\left(\varphi \bar{u}_{\varepsilon}\right)-\int_{\Omega} u_{\varepsilon} \sigma_{\varepsilon} \cdot \nabla \varphi-\int_{\Omega} f \varphi\left(\bar{u}_{\varepsilon}-u_{\varepsilon}\right),
$$

which gives

$$
\limsup _{\varepsilon \rightarrow 0} \int_{\Omega} \varphi \sigma_{\varepsilon} \cdot \nabla u_{\varepsilon} \leq \int_{\Omega} a_{0}\left(\nabla u_{0}\right) \cdot \nabla u_{0}
$$

The latter, combined with (3.22), implies

$$
\sigma_{\varepsilon} \cdot \nabla u_{\varepsilon} \rightarrow a_{0}\left(\nabla u_{0}\right) \cdot \nabla u_{0} \text { in } \mathcal{D}^{\prime}(\Omega) .
$$

Since $K_{\varepsilon} \rightarrow K_{0}$ in the Mosco sense, then taking $v_{0}=u_{0}$ in (3.23), we get

$$
\begin{aligned}
\int_{\Omega} a_{0}\left(\nabla u_{0}\right) \cdot \nabla u_{0} & \geq \limsup _{\varepsilon \rightarrow 0} \int_{\Omega} \sigma_{\varepsilon} \cdot \nabla u_{\varepsilon} \\
& \geq \liminf _{\varepsilon \rightarrow 0} \int_{\Omega} \sigma_{\varepsilon} \cdot \nabla u_{\varepsilon} \\
& \geq \int_{\Omega} \varphi a_{0}\left(\nabla u_{0}\right) \cdot \nabla u_{0},
\end{aligned}
$$

and letting $\varphi \rightarrow 1$, we obtain (3.24).

Remark 3.2. If in (1.8) we have $f_{\varepsilon}$ instead of $f$ and $f_{\varepsilon} \rightarrow f$ in $L^{s}(\Omega)$, then the conclusion of the Theorem 3.1 still holds.

Remark 3.3. Since there are Lewy-Stampacchia inequalities also for the two obstacles problem (see [19]), the Theorem 3.1 can be extended for two obstacles problems with similar assumptions.

\section{Convergence of the Coincidence sets}

In this section, using the Lewy-Stampacchia inequalities, we prove a stability result for the coincidence sets as it was done, for example, in Theorem 6:6.1 in [18].

Theorem 4.1. Let the conditions of Theorem 3.1 hold. If, as $\varepsilon \rightarrow 0$,

$$
\begin{aligned}
& u_{\varepsilon}-\psi_{\varepsilon} \rightarrow u_{0}-\psi_{0} \quad \text { in } L^{1}(\Omega), \\
&\left(A_{\varepsilon} \psi_{\varepsilon}-f\right)^{+} \rightarrow\left(A_{0} \psi_{0}-f\right)^{+} \text {in } L^{1}(\Omega), \\
& A_{\varepsilon} u_{\varepsilon} \rightarrow A_{0} u_{0} \quad \text { in } \mathcal{D}^{\prime}(\Omega), \\
& \int_{S} d\left(A_{0} \psi_{0}-f\right) \neq 0, \forall S \subset \Omega \text { such that }|S|>0,
\end{aligned}
$$

and

$$
A_{0} u_{0}-f=\left(A_{0} \psi_{0}-f\right) \chi_{0} \text { a.e. in } \Omega
$$


where $\chi_{0}$ is the characteristic function of the set $I_{0}:=\left\{u_{0}=\psi_{0}\right\}$, then the coincidence sets $I_{\varepsilon}:=\left\{u_{\varepsilon}=\psi_{\varepsilon}\right\}$ converge in measure, i.e.,

$$
\chi_{\varepsilon} \rightarrow \chi_{0} \text { in } L^{p}(\Omega), \forall p \in[1, \infty),
$$

where $\chi_{\varepsilon}$ is the characteristic function of $I_{\varepsilon}$.

Proof. From the Lewy-Stampacchia inequalities we have

$$
f \leq A_{\varepsilon} u_{\varepsilon} \leq f+\left(A_{\varepsilon} \psi_{\varepsilon}-f\right)^{+} \text {a.e. in } \Omega .
$$

Hence, there exists a function $q_{\varepsilon} \in L^{\infty}(\Omega)$, such that,

$$
A_{\varepsilon} u_{\varepsilon}-f=q_{\varepsilon}\left(A_{\varepsilon} \psi_{\varepsilon}-f\right)^{+} \text {a.e. in } \Omega \text {, }
$$

and

$$
0 \leq q_{\varepsilon} \leq \chi_{\varepsilon} \leq 1 \text { a.e. in } \Omega .
$$

Then for a subsequence (still denoted by $\varepsilon$ ), one has

$$
q_{\varepsilon} \rightarrow q \text { and } \chi_{\varepsilon} \rightarrow \chi_{*} \text { in } L^{\infty}(\Omega)-\text { weak* }^{*}
$$

for functions $q, \chi_{*} \in L^{\infty}(\Omega)$. The inequalities (4.7) imply

$$
0 \leq q \leq \chi_{*} \leq 1 \text { a.e. in } \Omega \text {. }
$$

Using (4.2), (4.3) and (4.8), we pass to the limit, as $\varepsilon \rightarrow 0$, in (4.6) and obtain

$$
A_{0} u_{0}-f=q\left(A_{0} \psi_{0}-f\right)^{+} \text {a.e. in } \Omega \text {. }
$$

The latter, combined with (4.5) provides

$$
q\left(A_{0} \psi_{0}-f\right)^{+}=\left(A_{0} \psi_{0}-f\right) \chi_{0} \text { a.e. in } \Omega .
$$

Note that in the region $\left\{A_{0} \psi_{0}>f\right\},(4.10)$ and (4.4) imply that $q=\chi_{0}$, while in $\left\{A_{0} \psi_{0} \leq f\right\}, \chi_{0}=0$. Therefore, $q \geq \chi_{0}$ a.e. in $\Omega$. Consequently, from (4.9) we get

$$
\chi_{0} \leq \chi_{*} \text { a.e. in } \Omega \text {. }
$$

On the other hand, from (4.1) and (4.8) one has

$$
0=\int_{\Omega} \chi_{\varepsilon}\left(u_{\varepsilon}-\psi_{\varepsilon}\right) \rightarrow \int_{\Omega} \chi_{*}\left(u_{0}-\psi_{0}\right)=0,
$$

thus $\chi_{*}\left(u_{0}-\psi_{0}\right)=0$ a.e. in $\Omega$. Consequently, if $u_{0}>\psi_{0}$, then $\chi_{*}=0$, and since $0 \leq \chi_{*} \leq 1$, one obtains

$$
\chi_{0} \geq \chi_{*} \text { a.e. in } \Omega .
$$

Therefore, $\chi_{0}=\chi_{*}$, and the whole sequence $\chi_{\varepsilon}$ converges to $\chi_{0}$ as $\varepsilon \rightarrow 0$, first weakly, and since they are characteristic functions, also strongly in any $L^{p}(\Omega)$, for any $p \in[1, \infty)$.

Remark 4.1. If $\psi_{0}=0$ and the right hand side is regular enough, the condition (4.5) holds automatically, since in this particular case one has porosity of the free boundary from [9] (hence, the free boundary has Lebesgue measure zero), which provides (4.5). 
Remark 4.2. The assumption (4.4) is a weaker version of the condition

$$
A_{0} \psi_{0}-f \neq 0 \text { a.e. in } \Omega \text {, when } A_{0} \psi_{0} \in L^{1}(\Omega) \text {. }
$$

Theorem 4.2. Let the conditions of Theorem 3.1 and also $s>n / 2$. If $\psi_{\varepsilon} \rightarrow \psi_{0}$, uniformly, $\left.\psi_{0}\right|_{\partial \Omega}<0$ and

$$
\overline{\operatorname{int}\left\{u_{0}=\psi_{0}\right\}}=\left\{u_{0}=\psi_{0}\right\}=I_{0},
$$

then the coincidence sets $I_{\varepsilon}:=\left\{u_{\varepsilon}=\psi_{\varepsilon}\right\}$ converge in the Hausdorff distance to $I_{0}$.

Proof. Using [13, Theorem 3.2], we obtain the uniform Hölder continuity of solutions. The uniform Hölder continuity of the obstacles then implies, as $\varepsilon \rightarrow 0$, the convergence $u_{\varepsilon} \rightarrow u_{0}$, uniformly in compact subsets of $\Omega$. This, in turn, provides the convergence of the coincidence sets in Hausdorff distance as in [8] and [18, Theorem 6:6.5].

Acknowledgments. This work was partially supported by FCT-Portugal grant SFRH/BPD/92717/2013.

\section{REFERENCES}

[1] H. Attouch and C. Picard, Variational inequalities with varying obstacles. The general form of the limit problem, J. Funct. Anal. 50 (1983), 329-386.

[2] L. Boccardo, Lewy-Stampacchia inequality in quasilinear unilateral problems and applications to the G-convergence, Boll. Unione Mat. Ital. (9) 4 (2011), 275-282.

[3] L. Boccardo and I.C. Dolcetta, G-convergenza e problema di Dirichlet unilaterale, Boll. Unione Mat. Ital. 4 (1975), 115-123.

[4] L. Boccardo and F. Murat, Nouveaux résultas de convergence dans des probèmes unilateraux, Research Notes in Math. 60 (1982), 64-85.

[5] L. Boccardo and F. Murat, Homogenization of nonlinear unilateral problems, Composite media and homogenization theory; an international center for theoretical physics workshop, Trieste, Italy, January 1990 (1991), 81-105.

[6] D. Cioranesco and P. Donato, An introduction to homogenization, Oxford lecture series in Mathematics and its applications 17, Oxford University Press Inc., New York, 1999.

[7] V.Chiado Piat, G. Dal Maso and A. Defranceschi, G-convergence of monotone operators, Ann. Inst. Henri Poincaré 7 (1990), 123-160.

[8] M. Codegone and J.F. Rodrigues, Convergence of the coincidence set in the homogenization of the obstacle problem, Ann. Fac. Sci. Toulouse Math. (5) 3 (1981), 275-285.

[9] S. Challal, A. Lyaghfouri, J.F. Rodrigues and R. Teymurazyan, On the regularity of the free boundary for quasilinear obstacle problems, Interfaces Free Bound. 16 (2014), 359-394.

[10] L. Diening, P. Harjulehto, P. Hästö and M. Ruzicka, Lebesgue and Sobolev spaces with variable exponents, Lecture Notes in Mathematics 2017 (2011).

[11] L.C. Evans, Weak convergence methods for nonlinear partial differential equations, American Mathematical Society, 1990.

[12] X. Fan and Q. Zhang, Existence of solutions for $p(x)$-Laplacian Dirichlet problem, Nonlinear Anal., Theory Methods Appl. 52 (2003), 1843-1852.

[13] X. Fan and D. Zhao, The quasi-minimizer of integral functionals with $m(x)$ growth condition, Nonlinear Anal. 39 (2000), 807-816. 
[14] X. Fan and D. Zhao, On the spaces $L^{p(x)}(\Omega)$ and $W^{m, p(x)}(\Omega)$, J. Math. Anal. Appl. 263 (2001), 424-446.

[15] D. Kinderlehrer and G. Stampacchia, An introduction to variational inequalities and their applications, SIAM Classics in Applied Mathematics 31, 2000, xxii+306p.

[16] F. Murat, Compacité par compensation, Ann. Scuola Norm. Sup. Pisa. Cl. Sci (4) 5 (1978), 489-507.

[17] A.A. Pankov, G-convergence and homogenization of nonlinear partial differential operators, Kluwer Academic Publishers, Dordrecht, 1997.

[18] J.F. Rodrigues, Obstacle problems in mathematical physics, North-Holland Mathematics Studies 134 (Notas de Matematica 114), Elsevier Science Publishers B.V., 1991.

[19] J.F. Rodrigues and R. Teymurazyan, On the two obstacles problem in Orlicz-Sobolev spaces and applications, Complex Var. Elliptic Equ. 56 (2011), 769-787.

[20] G.V. Sandrakov, Homogenization of variational inequalities for obstacle problems, Sbornik, Mathematics 196 (2005), 541-560.

[21] G.V. Sandrakov, Homogenization of nonlinear equations and variational inequalities with obstacles, Doklady Mathematics 73 (2006), 178-181.

[22] V.V. Zhikov, Lavrent'ev effect and the homogenization of nonlinear variational problems, Differ. Equations 27 (1991), 32-39.

[23] V.V. Zhikov and S.E. Pastukhova, On the compensated compactness principle, Doklady Mathematics 82 (2010), 590-595.

[24] V.V. Zhikov and S.E. Pastukhova, Homogenization of monotone operators under conditions of coercivity and growth of variable order, Mathematical Notes 90 (2011), 48-63.

Department of Mathematics, Federal University of Rio Grande do Sul, Av. Bento Gon calves 9500, 91509-900 Porto Alegre, RS, Brazil.

E-mail address: diego.marcon@ufrgs.br

CMafcio, Department of Mathematics, Faculty of Sciences, University of Lisbon, Portugal.

E-mail address: jfrodrigues@ciencias.ulisboa.pt

CMUC, Department of Mathematics, University of Coimbra, 3001-501 CoimBRA, Portugal.

E-mail address: rafayel@utexas.edu 